INPLASY

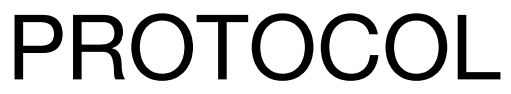

To cite: Lu et al. The clinical efficacy and safety of traditional Chinese medicine in the treatment of recurrent aphthous stomatitis: A protocol of systematic review and meta-analysis. Inplasy protocol 202080126. doi: 10.37766/inplasy2020.8.0126

Received: 31 August 2020

Published: 31 August 2020

Corresponding author: Jiaping Lu

natalieljp@126.com

Author Affiliation:

Department of Stomatology,

Shanghai Xuhui District Dental

Center, Shanghai, China

Support: ZK2019B12;

SHXYF201908

Review Stage at time of this submission: The review has not yet started.

Conflicts of interest:

All authors declare that they have no conflict of interest.

\section{The clinical efficacy and safety of traditional Chinese medicine in the treatment of recurrent aphthous stomatitis: A protocol of systematic review and meta-analysis}

Lu, J1; Zhang, N2; Qin, W³.

Review question / Objective: P: diagnosed as symptomatic recurrent aphthous stomatitis; I: traditional Chinese medicine; C: control groups were other treatment; O: clinical efficacy, pain relief, duration of wound healing, effect on wound healing, rate of recurrence, adverse events and safety; S: comparative design were included.

Condition being studied: Recurrent aphthous stomatitis.

Information sources: We will perform literature searches using the following electronic bibliographic databases from their inception onwards to the August 2020: MEDLINE, Springer, Web of Science, PubMed, EMBASE, the Cochrane Central Register of Controlled Trials, Evidence Based Medicine Reviews, VIP, and CNKI. We will not establish any limitations to language and publication status. The following electronic databases were searched from their inception dates through August 2020.

INPLASY registration number: This protocol was registered with the International Platform of Registered Systematic Review and Meta-Analysis Protocols (INPLASY) on 31 August 2020 and was last updated on 31 August 2020 (registration number INPLASY202080126).

\section{INTRODUCTION}

Review question / Objective: P: diagnosed as symptomatic recurrent aphthous stomatitis; I: traditional Chinese medicine; C: control groups were other treatment; $O$ : clinical efficacy, pain relief, duration of wound healing, effect on wound healing, rate of recurrence, adverse events and safety; S: comparative design were included. 
Condition being studied: Recurrent aphthous stomatitis.

\section{METHODS}

Participant or population: Patients were diagnosed as symptomatic recurrent aphthous stomatitis.

Intervention: Traditional Chinese medicine.

\section{Comparator: Other treatment.}

Study designs to be included: Comparative design.

Eligibility criteria: We will include all studies comparing the TCM in the treatment of RAS, including observational study and RCT.

Information sources: We will perform literature searches using the following electronic bibliographic databases from their inception onwards to the August 2020: MEDLINE, Springer, Web of Science, PubMed, EMBASE, the Cochrane Central Register of Controlled Trials, Evidence Based Medicine Reviews, VIP, and CNKI. We will not establish any limitations to language and publication status. The following electronic databases were searched from their inception dates through August 2020.

Main outcome(s): The criterion efficacy will be divided into four categories: complete remission (CR), partial remission (PR), stable (SD) and invalid (PD). The total eff e ctive rate was (CR+PR)/ $(C R+P R+S D+P D) \times 100 \%$.

Additional outcome(s): (1) pain relief (immediate and during healing); (2) duration of wound healing; (3) effect on wound healing; (4) rate of recurrence; (5) adverse events; (6) safety.

Quality assessment / Risk of bias analysis: The Cochrane risk of bias tool, which is recommended by the Cochrane Reviewer's Handbook 5.0.2, will be used to evaluate the quality of the included studies. Two independent reviewers will evaluate the quality of selected articles from the following 5 aspects: selection bias (random sequence generation or allocation concealment), performance bias and detection bias (blinding), attrition bias (incomplete outcome data), reporting bias (selective outcome reporting), and other biases. If necessary, we will contact the corresponding author to clarify issues. The result of the consistency evaluation will be presented with Kappa statistics, Kappa value $<0.75$ will be considered the consistency have reached. Any disagreements will be resolved through discussion or consultation.

Strategy of data synthesis: We will undertake RevMan $\mathbf{5 . 3}$ software to analyze data and to perform meta-analysis if it is necessary. We will calculate all continuous data using mean difference or standardized mean difference and $95 \%$ confidence intervals. As for dichotomous data, we will exert it using risk ratio and $95 \% \mathrm{Cl}$. The heterogeneity as determined by the

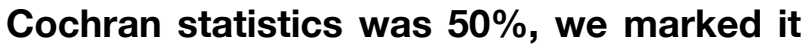
as a considerable level of heterogeneity; otherwise, we considered it to be a good homogeneity. We also assessed clinical heterogeneity. Statistically and clinically homogeneous studies were pooled using a fixed-effects model; otherwise, a randomeffects model was used when the heterogeneity was significant. Additionally, subgroup analysis will be operated to explore any possible reasons for the high heterogeneity. Whenever it is possible, we will conduct meta-analysis if at least 3 eligible criteria are fulfilled. Otherwise, meta-analysis will not be carried out if only 1 or 2 studies meet the inclusion criteria. Under such situation, the findings will be presented in a narrative summary. We will perform narrative synthesis if running meta-analysis is inappropriate due to the high heterogeneity. All narrative descriptions will be carried out based on the Guidance on the Conduct of Narrative Synthesis in Systematic Reviews.

Subgroup analysis: Based on available data, we will perform the following subgroup analyses: different types of treatment. 
Sensibility analysis: We will consider running sensitivity analysis to identify the robustness and stability of merged results by excluding studies with high risk of bias.

Country(ies) involved: China.

Keywords: protocol; systematic review; traditional Chinese medicine; recurrent aphthous stomatitis.

Contributions of each author:

Author 1 - Jiaping Lu.

Author 2 - Naizhen Zhang.

Author 3 - Wenhao Qin. 